\title{
Acceptability of chatbot versus General Practitioner consultations for healthcare conditions varying in terms of perceived stigma and severity (Preprint)
}

Oliver Miles

\begin{abstract}
Aims: This study aimed to assess how perceived stigma and severity of health issues are associated with the acceptability for a health consultation source: i) a chatbot, ii) a General Practitioner (GP) or iii) a GP-chatbot combination. Methods: Between May and June 2019, an online study, advertised via Facebook, was completed by a convenience sample of 237 participants from the UK. The design was an online factorial simulation experiment with three within-subject factors (health issue stigma, health issue severity and consultation source) and six betweensubject covariates resulting in 12 conditions where participants rated acceptability of each consultation source for each health condition. Both research questions were analysed with a single mixed-model analysis of variance (ANOVA). Results: More severe health issues decreased the acceptability for chatbots as a consultation source $F(2,372)=118.14, p<.001$, partial $\eta 2=0.388$, while more stigmatised health issues increased the acceptability for chatbots as a consultation source $F(2,372)=$ 12.99, $\mathrm{p}<.001$, partial $\eta 2=0.65$. There was no significant association between participants' characteristics and acceptability of consultation sources. Conclusions: Chatbots may be more acceptable for consultations regarding more stigmatised health and less acceptable for conditions of higher perceived severity.
\end{abstract}

\section{Definitions}

Acceptability

Defined by Mandeep Sekhon

Chatbots

Defined by Adam Palanica

1. Introduction 
Chatbots are programmes designed to simulate human conversation via text or speech (Palanica, Flaschner, Thommandram, Li, \& Fossat, 2019). Artificial Intelligence (AI) can be implemented in the form of chatbots to triage or diagnose a patient's health issues ( $\mathrm{Yu}$, Beam, \& Kohane, 2018). However, little is known about acceptability of chatbots to patients. A variety of factors may influence this acceptability, including perceived stigma and severity of the presenting symptoms. This study examined whether perceived stigma and severity of various health issues influenced chatbot acceptability as a consultation source compared to a General Practitioner (GP) and a GP-chatbot combination. It also assessed whether participant characteristics influenced chatbot acceptability.

\subsection{Benefits of chatbots}

One example of a chatbot is the Babylon "GP at Hand" (GP at Hand) service. This incorporates chatbot functionality to triage patient's health issues and direct them to relevant modes of care (Armstrong, 2018). A study by Razzaki et al., compared the diagnoses of health conditions by doctors and the 'GP at Hand' service. This chatbot was able to diagnose the conditions with accuracy comparable to human doctors. A recent report by the (Hammersmith and Fulham Clinical Commissioning Group, 2018) concluded that the GP at Hand service is able to address barriers to health equality experienced by people in conventional GP settings - such as an ease of access to primary healthcare for those with reduced mobility. As a result, chatbots are receiving increasing attention as a technology that can benefit and innovate healthcare systems (Garg, Williams, Ip, \& Dicker, 2018; Hamet \& T remblay, 2017; He et al., 2019; Jiang et al., 2017; Vaidyam, Wisniewski, Halamka, Kashavan, \& Torous, 2019).

\subsection{Acceptability}

Acceptability of healthcare interventions is defined as the extent to which people delivering or receiving the intervention consider it to be appropriate based on anticipated or experiential cognitive and emotional responses to the intervention (Sekhon, Cartwright, \& Francis, 2017). Using this theoretical framework, acceptability is assumed to encompass all motivational factors that influence a person's intention to use a chatbot for health issue diagnosis. This includes both reflective (e.g. intention) and automatic motivational (e.g. emotional responses) processes that influence a person's usage behaviour (Michie, van Stralen, \& West, 2011). Hence, acceptability has implications for actual behavioural usage of the technology.

\subsection{Perception of chatbots for health issue diagnosis}


In healthcare, chatbots that are implemented as diagnostic consultation sources can be presented with a wide variety of health issues by users. It is unlikely that people will be as accepting of being diagnosed by a chatbot for health issues, especially health issues that are severe. For severe health issues, chatbot acceptability may be lower due to a number of concerns. First of all, the issue of chatbot responsibility and liability (Luxton, 2016; Meskó, 2017; Vaidyam et al., 2019) - who takes the blame if the chatbot misdiagnoses someone? Secondly, chatbot competence - are chatbots competent enough to diagnose humans correctly? (Luxton, 2016; Nadarzynski, Miles, Cowie, \& Ridge, 2019; Palanica et al., 2019); and, thirdly, patient safety (Luxton, 2016) - are chatbots adhering to patient safety protocols and standards?

In the case of stigmatised health issues, research has found that people are more willing to disclose sensitive health information to chatbots in comparison to health professionals (Lucas, Gratch, King, \& Morency, 2014; Lucas et al., 2017). Nadarzynski et al. (2019) demonstrated that chatbot acceptability may be higher for stigmatised health issues as they offer greater anonymity. Users may be more accepting of a diagnosis from a chatbot for more stig matised health issues than severe health issues.

\section{Research objectives}

Due to the accessibility of chatbots it is likely that there will be many different user demographics utilising the services. Although there has been little research into acceptability, research has found that attitudes and usage of technology are influenced by factors such as prior chatbot and confidence in chatbot knowledge (Laumer, Maier, \& Gubler, 2019), age (Nadarzynski et al., 2019), gender, educational level and average time spent on the internet each week (Kontos, Blake, Chou, \& Prestin, 2014). Fadhil (2018) suggested a way to mitigate the acceptance of chatbots as a consultation source is to utilise a GP-chatbot combination. This is where chatbots triage patients and manage minor health issues, while GPs could deal with more serious health issues and have their diagnoses validated by Al.

The current study addresses the following research questions:

1. Does the perceived severity of a presenting condition reduce the acceptability of use of a chatbot or a chatbot-GP combination for an initial consultation compared with a GP?

2. Does the perceived stigma of a presenting condition increase the acceptability of use of a chatbot or a chatbot-GP combination for an initial consultation compared with a 
GP?

3. To what extent do participant characteristics influence acceptability of chatbot, chatbot-GP combination versus GP?

\section{Methodology}

The study was an online factorial simulation experiment design with three within-subject factors and six between-subject covariates. The within-subject factors were the stigma and the severity of the health issue and the consultation source. The between-subject covariates were prior chatbot knowledge, confidence of chatbot knowledge, average internet usage, age, gender and education level. The outcome variable was chatbot acceptability. The study protocol and analysis plan were pre-reg istered on the Open Science Framework (https://osf.io/szgma/). Approval was granted by the ethics committee of University College London, UK (14917/001).

\subsection{Participants and recruitment}

Due to cost and practical reasons, all adults with an access to the Internet, as potential users of chatbots, living in the UK, were invited to participate. Between May 2019 to June 2019, potential participants were recruited through Facebook advertisement with a link to an online survey. The incentive was a 10 pence donation to charity per participant upon study completion. There were no specific exclusion criteria.

\subsection{Measures}

The outcome measure, acceptability for each consultation source, was based on the acceptability framework proposed by Sekhon et al. (2017) and was operationalised as a proxy measure 'willingness'. Participants were presented with the statement 'I would be willing to use this option to find out what is wrong and recommend treatment' and were asked to rate their willingness to use each of the three consultation sources on a 5-point Likert scale (rang ing from 1 = 'not very willing at all'; to 5 = 'very willing') for each presented health issue. Scores under 3 were interpreted as a less acceptable rating by participants, while scores over 3 were interpreted as a more acceptable rating by participants.

Prior chatbot knowledge was measured by asking participants 'Have you used a chatbot before? Unsure (coded 0), yes (i), no (ii)'. While confidence in chatbot knowledge was measured by asking participants 'Do you feel confident that you know what a chatbot is?' This was rated on a 5-point Likert-scale by participants ranging from 1 = 'Not very confident at all' to 5 = 'very confident'. 
Internet usage was measured using questions adapted from the Organisation for Economic Cooperation and Development (OECD) ICT Access and Usage by Households and Individuals questionnaire (OECD, 2015). While, demographic questions measuring age, gender, and educational attainment were adapted from the Government Statistical Service (GSS) educational attainment and national harmonised demographic questionnaires (GSS, 2004, 2017).

\section{Experimental conditions}

The three experimental factors were: i) health issue stigma (more/ less), ii) health issue severity (high/ low) and iii) consultation source (chatbot, GP, GP-chatbot combination). This resulted in 12 conditions: 1) less stigmatised, low severity, chatbot; 2) less stig matised, low severity, GP; 3) less stigmatised, low severity, GP-chatbot combination; 4) more stigmatised, low severity, chatbot; 5) more stigmatised, low severity, GP; 6) more stigmatised, low severity, GP-chatbot combination; 7) less stigmatised, high severity, chatbot; 8) less stigmatised, high severity, GP; 9) less stigmatised, high severity, GP-chatbot combination; 10) more stigmatised, high severity, chatbot; 11) more stigmatised, high severity, GP; 12) more stigmatised, high severity, GP-chatbot combination.

The participants were presented with three health issues for each high/less stigmatised or high/low severity condition. They were asked to rate their acceptability of each of the three consultation sources for each experimental factor. In total, participants were asked to complete 36 acceptability ratings (three for each condition) and were blinded to the predicted stigma or severity of the health issue. This was to mitigate for the possibility that the presented health issue's stigma or severity may be interpreted differently.

The health issues were identified and selected via a pilot study consisting of 37 participants using convenience sampling. Participants rated their perceived stigma and severity of 40 health issues, identified from the NHS's Health A to Z directory (NHS, 2019), on a five-point Likert scale ( 1 = low perceived sigma/ severity, 5 = high perceived stigma or severity). As per consensus from the research team, mean scores under two were rated as low perceived stigma or severity and mean scores over 3.5 were rated as high perceived sigma or severity.

\subsection{Procedure}

Patients were presented with the information page and asked to consent to the survey. Participants were then asked about their knowledge of chatbots and were presented with 
a video and text transcript describing a chatbot (https://www.youtube.com/watch? v=WRcCSUQJasW) to maximise engagement. Once participants had rated their confidence in chatbot knowledge, they were presented with the 12 health issues and asked to indicate the consultation source acceptability accordingly. Participants were then asked remaining demographic and behavioural questions.

\subsection{Statistical analyses}

The means (M) and standard errors (SE) of acceptability ratings for chatbot, GP and GPchatbot combination were calculated for all categories of participants where comparisons were being made.

Both research questions were addressed together in a single mixed-model analysis of variance (ANOVA). The model included all main effects and all 2-way interactions involving consultation source. It also included the 3-way interaction between apparent stigma, severity and consultation source. Acceptability scores over 3 were interpreted as more acceptable ratings of consultations scores, while scores under 3 were interpreted as less acceptable ratings of consultation source.

\section{Results}

A total of 237 participants completed the study (T able 1). The majority of the sample were female $(73.4 \%$, ), aged over 45 years old $(65.0 \%$, , and educated with a degree or higher (54.9\%). Most participants had no prior knowledge of chatbots (59.5\%) but were confident that they knew what a chatbot was once the concept was explained to them (70.9\%). Overall GPs were reported as the most acceptable consultation source, followed by a chatbot-GP combination and then chatbot (T able 2 ).

The interaction between the severity of the health issues and acceptability of the different consultation sources were found to be significant (T able 2). GPs and the GPchatbot combination were found to be more acceptable than chatbots for more severe health issues but not the less severe ones.

There was a significant interaction between the level of stigma of the health issue and the acceptability of the different consultation sources. The greater acceptability for GPs over chatbots was attenuated in the high stigma conditions (Table 2).

There was a significant three-way interaction between the stigma, the severity of the health issue and the acceptability of the different consultation sources (Table 2). The differences between the different sources were only evidence in the high severity conditions. In the low severity conditions, there was no clear evidence that participants 
had a preference.

None of the participant characteristics significantly influenced the acceptability ratings (Table 3).

\section{Discussion}

The results showed that for health conditions with low perceived severity chatbots, GPs or chatbot-GP combinations were judged to be approximately equally acceptable. However, for conditions of high perceived severity GPs were judged more acceptable than a chatbot-GP combination which was judged more acceptable that chatbots alone, with this difference between attenuated in conditions with high stigma. There was no clear evidence that participant characteristics influenced acceptability ratings.

\subsection{Participant acceptability of chatbots with severe health issues}

As expected, participants were less accepting of using a chatbot to diagnose high severity health issues. This may be a consequence of factors such as responsibility and liability (Luxton, 2016; Meskó, 2017; Vaidyam et al., 2019), chatbot competence (Luxton, 2016; Nadarzynski et al., 2019; Palanica et al., 2019), and patient safety (Luxton, 2016) discussed previously. It is possible that people may just want to deal with health professionals at time of distress.

\subsection{Participant acceptability of chatbots with stigmatised health issues} More stigmatised heath issues were found to increase how accepting a person is of using a chatbot as a consultation source if the severity of the health issue is low. This finding complements existing research into chatbots and stigmatised health issues (Cameron et al., 2017; Lucas et al., 2014) and provides evidence that chatbots may be a viable method of consultation for more stigmatised health issues. However, considering that acceptance of chatbots as a consultation source is low for more stigmatised/high severity health issues, it is apparent that severity of a health issue is a more important determinant of acceptability than stigma.

\subsection{Participant acceptability of a GP-chatbot combination}

As argued by Fadhil (2018a), a GP-chatbot combination did increase the chatbot acceptability. It is interesting that participants were not more accepting of a GP-chatbot combination. One would expect that a GP-chatbot combination would be the most acceptable consultation source as patients are receiving the same benefits as seeing a GP about their health issues, with the added benefit of triage and validation by chatbots 
and Al. The low acceptance scores could be explained by the neg ativity effect (Reeder \& Brewer, 1979); this is where negative attitudes are weighted more than positive attitudes when forming an evaluation.

\subsection{Implications of results}

\subsubsection{Implications of results on previous and current research}

These results build on existing evidence that the acceptability of chatbots in healthcare (Grolleman, van Dijk, Nijholt, \& van Emst, 2006; Laumer et al., 2019; Nadarzynski et al., 2019) and triangulates the findings of the existing qualitative research into healthcare chatbot acceptability by Laumer et al. (2019) and Nadarzynski et al. (2019). The results also inform the behaviour change discipline as acceptability can both be seen as an influence on technology uptake behaviour and as a criterion for selecting delivery options for a behaviour change intervention (Michie et al., 2011; Pereira \& Díaz, 2019).

\subsubsection{Implications of results on theory development}

During the undertaking of this study, the Acceptability Model for Conversational Agent Disease Diagnosis was developed by Laumer et al. (2019). This model was based upon the Unified Theory of Acceptance and Use of technology for the consumer context (UTAUT2) (Venkatesh, Thong, \& Xu, 2012) and was adapted to explain patients' adoption of chatbots in the private healthcare context using qualitative analysis. The model proposed 13 predictors of intention to voluntarily use a chatbot in healthcare which are assumed as constructs of acceptability such as. Considering the results in this study, how accepting people are of chatbots in healthcare is influenced by specific healthcare related determinates such as the stigma and severity of health issues. Patients in the healthcare context cannot be assumed to make voluntary decisions similarly to consumers regardless if the healthcare is private or public. Due to a specific health issue a person may have, decision making to accept and utilise a chatbot in the healthcare context is bounded in rationality (Simon, 1990) far more than a conventional decision-making context. The failure to account for specific healthcare related determinates of acceptability is a significant limitation of the model proposed by Laumer et al. (2019).

\subsubsection{Implications of results on the healthcare industry}

This study has implications for the healthcare industry as the results increase the understanding of where chatbots would be most acceptable for patients to use if implemented. It is possible that there is a lack of acceptance of chatbots, particularly in the UK, because there is a lack of a perceived need for the technology. Indeed, there is a perception that chatbots will replace humans in jobs and increase unemployment (Meskó, 
2017). By implementing chatbots into areas where they are more likely to be accepted, for example as consultation source for more stigmatised or less severe health issues. As more people use and accept chatbots in these areas, it may inadvertently increase acceptance as people start to perceive the need for the technology (Rogers, 1962).

\subsection{Limitations}

The study had several limitations. As this study was advertised online, people who use the internet regularly are more likely to be exposed to the study advert. Consequently, the population may have resulted in a more technology accepting population participating in the study due to self-selection bias. Secondly, the amount of excluded cases equated to almost $1 / 3$ of the total participants. Therefore, there is a risk of bias due to this missing data (assuming it is not missing-at-random). Therefore, reducing the population validity of the study. This may explain why there was insufficient evidence to conclude that participant characteristics influenced the acceptability ratings of the different consultation sources.

Regarding the study's measures, the self-report of willing ness is not real-life behaviour enactment. Willingness is a measure of intention; there is a well-established gap between an intention to do a behaviour an actual behaviour enactment (Bhattacherjee \& Sanford, 2009; Sheeran \& Webb, 2016). This study's results may not provide an accurate representation of how acceptability influences a person's usage of a chatbot in healthcare. This limitation exists due to a lack of a valid measure of acceptability.

This was a simulation study; the conditions participants undertook were not real situations, therefore implicating the predictive validity of these results. If a person has or has experienced a specific health issue, they may be more/less accepting of using a chatbot as a consultation source than if they imagine the health issue. The health issues used in this study may not be accurate representations of more/less stigmatised or high/ low severity health issues. Hence, the face validity of this experiment may be reduced. For example, those that have health anxiety perceive health issues with greater severity (NHS, 2017). This study may have found different acceptability ratings with different health issues.

\subsection{Suggestions for future research}

Considering the discussion, future theoretical development needs to consider how specific health issues influence the acceptability of chatbots for disease diagnosis. For example, the decision to accept a chatbot for diagnosis may be influenced by factors such as the urgency for diagnosis, the person's wellbeing and how the individual feels 
about their symptoms. Once factors like this are considered, then a more comprehensive model of chatbot acceptability in healthcare can be developed.

Considering the limitations, future research must prioritise the development of a valid measure of acceptability for chatbots in healthcare to increase the validity of any future findings. Studies looking into how health issues influence acceptability must be undertaken with a broader sample, including different participants, and a broader set of health issues to assess if they influence acceptance levels of chatbots in healthcare.

\section{Conclusion}

In a simulation experiment of acceptability of chatbots for healthcare consultations, their acceptability relative to GP consultations was reduced for symptoms with high judged severity and increased for ones with higher judged stigma. Acceptability did not appear to be influenced by participant demographic characteristics or experience with chatbots. Overall, this study has broadened the understanding of the acceptability of chatbots in healthcare, moving insight from a general overview to how specific health issues influence the acceptability of chatbots as consultation sources. This has implications for where chatbots are best implemented into healthcare and for understanding the motivations behind chatbot use in healthcare. Future research needs to focus on more specific influences of the acceptability of chatbots in healthcare to develop a more holistic understanding. 


\begin{tabular}{|c|c|}
\hline Table 1 Sample character & \\
\hline Participant Characteristic & Total of the sample (\%) \\
\hline \multicolumn{2}{|l|}{ Gender } \\
\hline Male & $51(21.5)$ \\
\hline Female & $174(73.4)$ \\
\hline Missing & $12(5.1)$ \\
\hline \multicolumn{2}{|l|}{ Age } \\
\hline $18-44$ & $76(32.1)$ \\
\hline $45+$ & $154(65)$ \\
\hline Missing & $7(3)$ \\
\hline \multicolumn{2}{|l|}{ Educational level } \\
\hline Anything else & $99(41.8)$ \\
\hline Degree and above & $130(54.9)$ \\
\hline Missing & $8(3.4)$ \\
\hline \multicolumn{2}{|l|}{ Prior knowledge } \\
\hline No & 141 (59.5) \\
\hline
\end{tabular}




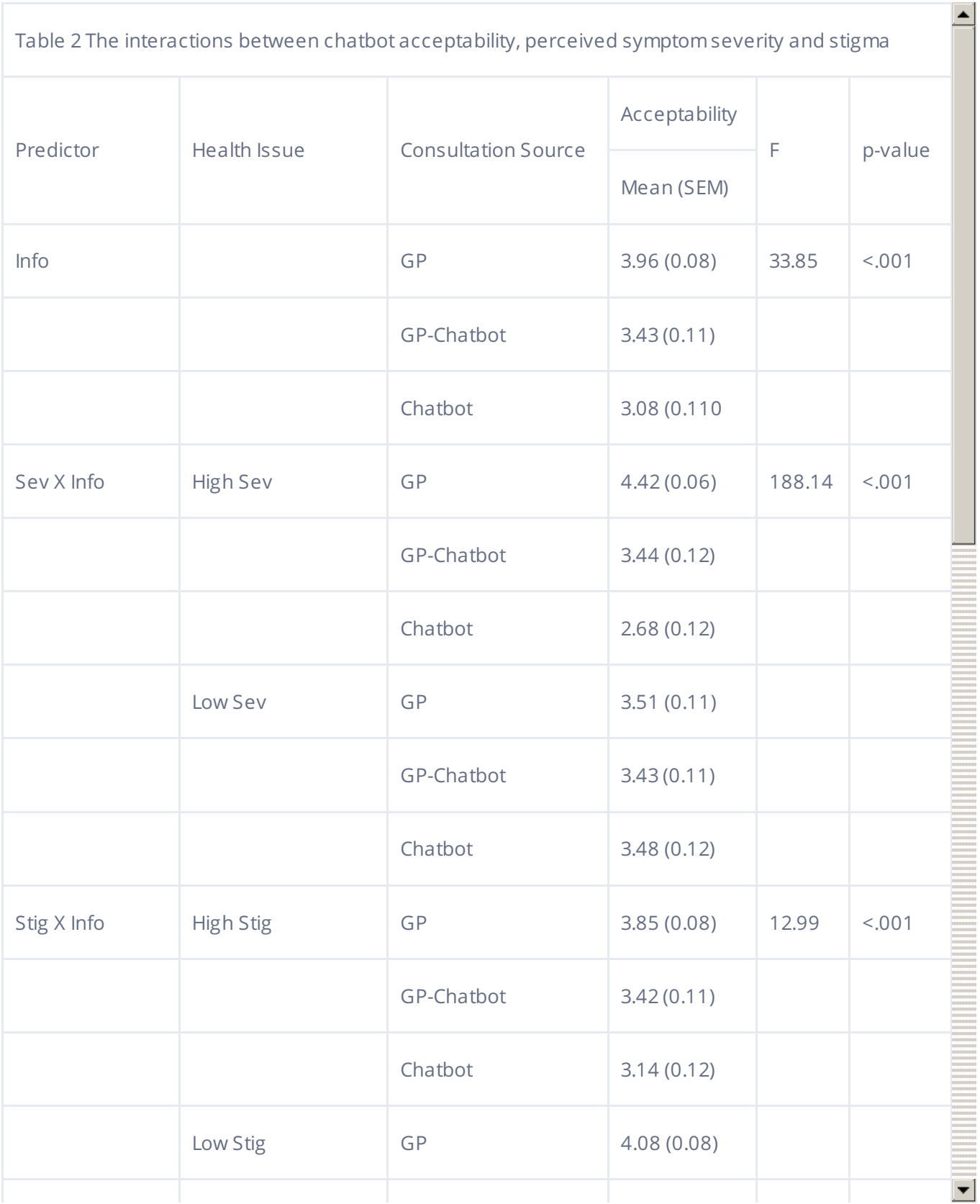




\begin{tabular}{|c|c|}
\hline Participant Characteristic & Acceptability Mean (SEM) \\
\hline \multicolumn{2}{|l|}{ Gender } \\
\hline Male & $3.61(0.12)$ \\
\hline Female & $3.38(0.08)$ \\
\hline \multicolumn{2}{|l|}{ Age } \\
\hline $18-44$ & $3.49(0.12)$ \\
\hline $45+$ & $3.50(0.09)$ \\
\hline \multicolumn{2}{|l|}{ Educational level } \\
\hline Anything else & $3.38(0.10)$ \\
\hline Degree and above & $3.60(0.09)$ \\
\hline \multicolumn{2}{|l|}{ Prior knowledge } \\
\hline No & $3.57(0.10)$ \\
\hline Yes & $3.41(0.11)$ \\
\hline Confidence in chatbot knowledge & \\
\hline Low confidence & $3.42(0.13)$ \\
\hline
\end{tabular}

\section{References}

1. Armstrong, S. (2018). The apps attempting to transfer NHS 111 online. BMJ, k156. https://doi.org/10.1136/bmj.k156

2. Bhattacherjee, A., \& Sanford, C. (2009). The intention-behaviour gap in technology usage: The moderating role of attitude strength. Behaviour \& Information Technology, 28(4), 389-401. https://doi.org/10.1080/01449290802121230

3. Cameron, G., Cameron, D., Megaw, G., Bond, R., Mulvenna, M., O'Neill, S., ... McT ear, M. (2017, July 1). Towards a chatbot for digital counselling. Presented at the 
Proceedings of the 31st International BCS Human Computer Interaction Conference (HCl 2017). https://doi.org/10.14236/ewic/HCl2017.24

4. Fadhil, A. (2018). A Conversational Interface to Improve Medication Adherence: Towards Al Support in Patient's Treatment. ArXiv:1803.09844 [Cs]. Retrieved from http://arxiv.org/abs/1803.09844

5. Garg, S., Williams, N. L., Ip, A., \& Dicker, A. P. (2018). Clinical Integration of Digital Solutions in Health Care: An Overview of the Current Landscape of Digital Technologies in Cancer Care. JCO Clinical Cancer Informatics, (2), 1-9. https://doi.org/10.1200/CCI.17.00159

6. Government Statistical Service. (2004). Education attainment and qualifications. Retrieved from https://gss.civilservice.gov.uk/guidances/harmonisation/0harmonised-principles/education-attainment-and-qualifications/\# annex-a

7. Government Statistical Service. (2017). Harmonised concepts and questions for social data sources. Retrieved from https://gss.civilservice.gov.uk/wpcontent/uploads/2016/03/Demographic-Info-June-17-Pending-informing-SPSC-1.pdf

8. Grolleman, J., van Dijk, B., Nijholt, A., \& van Emst, A. (2006). Break the Habit! Designing an e-Therapy Intervention Using a Virtual Coach in Aid of Smoking Cessation. In W. A. IJsselsteijn, Y. A. W. de Kort, C. Midden, B. Eggen, \& E. van den Hoven (Eds.), Persuasive Technology (Vol. 3962, pp. 133-141). https://doi.org/10.1007/11755494_19

9. Hamet, P., \& Tremblay, J. (2017). Artificial intelligence in medicine. Metabolism, 69, S36-S40. https://doi.org/10.1016/j.metabol.2017.01.011

10. Hammersmith and Fulham Clinical Commissioning Group. (2018). Equality and Health Inequalities Analysis: Standard Toolkit for CWHHE CCGs. Retrieved from https://www.hammersmithfulhamccg.nhs.uk/media/135838/PCCC-Item-6A-14August-2018-GPAH-EQIA.pdf

11. He, J., Baxter, S. L., Xu, J., Xu, J., Zhou, X., \& Zhang, K. (2019). The practical implementation of artificial intelligence technologies in medicine. Nature Medicine, 25(1), 30-36. https://doi.org/10.1038/s41591-018-0307-0

12. Jiang, F., Jiang, Y., Zhi, H., Dong, Y., Li, H., Ma, S., ... Wang, Y. (2017). Artificial intelligence in healthcare: Past, present and future. Stroke and Vascular Neurology, 2(4), 230-243. https://doi.org/10.1136/svn-2017-000101

13. Kontos, E., Blake, K. D., Chou, W.-Y. S., \& Prestin, A. (2014). Predictors of eHealth Usage: Insights on The Digital Divide From the Health Information National Trends Survey 2012. Journal of Medical Internet Research, 16(7), e172. https://doi.org/10.2196/jmir.3117

14. Laumer, S., Friedrich-Alexander, Maier, C., \& Gubler, F. (2019). CHATBOT ACCEPT ANCE IN HEALTHCARE: EXPLAINING USER ADOPTION OF CONVERSATIONAL AGENTS FOR 
DISEASE DIAGNOSIS. Proceedings of the 27th European Conference on Information Systems (ECIS).

15. Lucas, G. M., Gratch, J., King, A., \& Morency, L.-P. (2014). It's only a computer: Virtual humans increase willingness to disclose. Computers in Human Behavior, 37, 94-100. https://doi.org/10.1016/j.chb.2014.04.043

16. Lucas, G. M., Rizzo, A., Gratch, J., Scherer, S., Stratou, G., Boberg, J., \& Morency, L.-P. (2017). Reporting Mental Health Symptoms: Breaking Down Barriers to Care with Virtual Human Interviewers. Frontiers in Robotics and AI, 4, 51.

https://doi.org/10.3389/frobt.2017.00051

17. Luxton, D. D. (Ed.). (2016). Artificial intelligence in behavioral and mental health care. Amsterdam : Boston: Elsevier/Academic Press.

18. Meskó, B. (2017). The role of artificial intelligence in precision medicine. Expert Review of Precision Medicine and Drug Development, 2(5), 239-241. https://doi.org/10.1080/23808993.2017.1380516

19. Michie, S., van Stralen, M. M., \& West, R. (2011). The behaviour change wheel: A new method for characterising and designing behaviour change interventions. Implementation Science, 6(1), 42. https://doi.org/10.1186/1748-5908-6-42

20. Nadarzynski, T., Miles, O., Cowie, A., \& Ridge, D. (2019). Acceptability of artificial intelligence (AI)-led chatbot services in healthcare: A mixed-methods study. DIGITAL HEALTH, 5, 205520761987180. https://doi.org/10.1177/2055207619871808

21. National Health Service. (2017). Health Anxiety. Retrieved from https://www.nhs.uk/conditions/health-anxiety

22. National Health Service. (2019). Health A to Z. Retrieved from https://www.nhs.uk/conditions/

23. Organisation for Economic Cooperation and Development. (2015). The OECD model survey on ICT access and usage by households and individuals. Retrieved from https://www.oecd.org/sti/ieconomy/ICT -Model-Survey-Access-Usage-HouseholdsIndividuals.pdf

24. Palanica, A., Flaschner, P., Thommandram, A., Li, M., \& Fossat, Y. (2019). Physicians' Perceptions of Chatbots in Health Care: Cross-Sectional Web-Based Survey. Journal of Medical Internet Research, 21(4), e12887. https://doi.org/10.2196/12887

25. Pereira, J., \& Díaz, Ó. (2019). Using Health Chatbots for Behavior Change: A Mapping Study. Journal of Medical Systems, 43(5), 135. https://doi.org/10.1007/s10916-0191237-1

26. Reeder, G. D., \& Brewer, M. B. (1979). A schematic model of dispositional attribution in interpersonal perception. Psychological Review, 86(1), 61-79. https://doi.org/10.1037/0033-295X.86.1.61 
27. Rogers, E. (1962). Diffusion of innovations. Retrieved from https://teddykw2.files.wordpress.com/2012/07/everett-m-rogers-diffusion-ofinnovations.pdf

28. Sekhon, M., Cartwright, M., \& Francis, J. J. (2017). Acceptability of healthcare interventions: An overview of reviews and development of a theoretical framework. BMC Health Services Research, 17(1), 88. https://doi.org/10.1186/s12913-017-2031-8

29. Sheeran, P., \& Webb, T. (2016). The intention-behaviour gap. Social and Personality Psychology Compass, 10(9), 503-518. https://doi.org/10.1111/spc3.12265

30. Simon, H. A. (1990). Bounded Rationality. In J. Eatwell, M. Milgate, \& P. Newman (Eds.), Utility and Probability (pp. 15-18). https://doi.org/10.1007/978-1-349-20568-4_5

31. Vaidyam, A. N., Wisniewski, H., Halamka, J. D., Kashavan, M. S., \& Torous, J. B. (2019). Chatbots and Conversational Agents in Mental Health: A Review of the Psychiatric Landscape. The Canadian Journal of Psychiatry, 070674371982897. https://doi.org/10.1177/0706743719828977

32. Venkatesh, Thong, \& Xu. (2012). Consumer Acceptance and Use of Information Technology: Extending the Unified Theory of Acceptance and Use of Technology. MIS Quarterly, 36(1), 157. https://doi.org/10.2307/41410412

33. Yu, K.-H., Beam, A. L., \& Kohane, I. S. (2018). Artificial intelligence in healthcare. Nature Biomedical Engineering, 2(10), 719-731. https://doi.org/10.1038/s41551-018-0305-8 